\title{
Gonadal-Hypothalamic Interaction in Prepubertal and Pubertal Man: Effect of Clomiphene Citrate on Urinary Follicle-Stimulating Hormone and Luteinizing Hormone and Plasma Testosterone
}

\author{
Howard E. Kulin ${ }^{[30]}$, Melvin M. Grumbach, and Selna L. Kaplan \\ Department of Pediatrics, University of California Medical Center, San Francisco, California, USA
}

\begin{abstract}
Extract
The effect of administering various doses of clomiphene citrate upon gonadotropin excretion and plasma testosterone in 13 prepubertal and 13 pubertal patients of both sexes was evaluated. Follicle-stimulating hormone (FSH) and luteinizing hormone $(\mathrm{LH})$ were measured by the radioimmunoassay of urinary kaolin-acetone extracts. Clomiphene suppressed FSH excretion in two of five prepubertal patients given $0.1 \mathrm{mg} /$ day for 1 week. There were neither stimulatory nor suppressive effects when 0.01 or $0.001 \mathrm{mg} /$ day of the drug was administered.

Urinary gonadotropins and plasma testosterone decreased when $100 \mathrm{mg} / \mathrm{day}$ of clomiphene were administered to early pubertal individuals. Neither stimulation nor suppression took place when $10 \mathrm{mg}, 1 \mathrm{mg}$, or $0.1 \mathrm{mg} /$ day were given to early pubertal patients. In two girls in midpuberty who received $100 \mathrm{mg} /$ day of clomiphene, an LH rise was observed, and both of these individuals experienced menarche 17-24 days after the beginning of drug ingestion. Sequential studies in a boy between early and mid puberty revealed a change in gonadotropin and testosterone results from suppression to no response when $100 \mathrm{mg} /$ day of clomiphene were given for 1 week.

The data are consistent with the hypothesis that the hypothalamus of the early pubertal child is sensitive to negative feedback by the weak estrogen, clomiphene. Not until midpuberty can stimulatory effects of clomiphene, similar to those obtained in the adult, be demonstrated.
\end{abstract}

\section{Speculation}

Presumably there is a marked change in the sensitivity of the hypothalamic-pituitary negative feedback centers to gonadal steroids during sexual maturation. Since the feedback set-point continues to decrease during puberty, the change in sensitivity may in fact begin early in childhood. Puberty might then be viewed as a continuum lasting several years, secondary sexual characteristics beginning only when a critical level of steadily increasing gonadotropin secretion is attained. 


\section{Introduction}

Previous studies from this laboratory [9] suggested that the initiation of puberty in man is associated with a change in the sensitivity of the gonadal-hypothalamic negative feedback mechanism. Thus, the pubertal gonadotropin rise may result from diminished effectiveness of low prepubertal concentrations of gonadal hormones in suppressing pituitary follicle-stimulating hormone (FSH) and luteinizing hormone (LH).

In an attempt to delineate further gonadal-hypothalamic relations in the pubertal as well as prepubertal child, we have administered a wide range of doses of the weakly estrogenic anti-estrogen, clomiphene citrate, to 26 patients. It was of particular interest to determine whether varying the dose of clomiphene could elicit a stimulatory gonadotropin response in the immature individual as has been demonstrated in the adult $[3,7]$. Since the dose of clomiphene which causes an increase in gonadotropins in adults seems to suppress these hormones in children [9], we attempted to determine when the change in response takes place. The results of the present study indicate that suppression persists into early puberty; not until sexual maturation is moderately advanced are stimulatory gonadotropin responses elicited with clomiphene.

\section{Methods}

Plasma testosterone was determined by a competitive protein-binding technique which allowed the reliable detection of $0.3 \mathrm{ng}$ hormone [2]. Urinary $\mathrm{FSH}$ and $\mathrm{LH}$ were measured by radioimmunoassay $[5,21]$.

Kaolin-acetone concentrates of urine were utilized in the radioimmunoassay $[6,11,24]$ and allowed the determination of very low levels of gonadotropin excretion. Additionally, such concentrates can be serially diluted, assuring parallelism of each unknown with the standard curve (2nd International Reference Preparation of Human Menopausal Gonadotropin-2nd IRP) of the $\mathrm{LH}$ and FSH radioimmunoassays. We have found that close attention must be paid to parallelism between unknown and standard since assay sensitivity may be different for crude preparations of unknown (e.g., unprocessed urine or unprocessed serum) and relatively highly purified pituitary and urinary extracts (Fig. 1).

A 90-ml aliquot of a 24-hr urine collection was used to prepare kaolin-acetone concentrates. This amount of urine allows the use of $90-\mathrm{ml}$ centrifuge tubes, but does not permit enough hormone to be extracted so that LH can be routinely measured in urine of prepubertal subjects by this means. Consequently, only urinary FSH and plasma testosterone were determined in prepubertal individuals, whereas urinary LH and FSH, as well as plasma testosterone, were measured in pubescent patients.

\section{Preparation of Urine for Use in the Radioimmu- noassay}

A modification of the Albert kaolin-acetone technique [l] was used. A 90-ml aliquot of a 24-hr urine

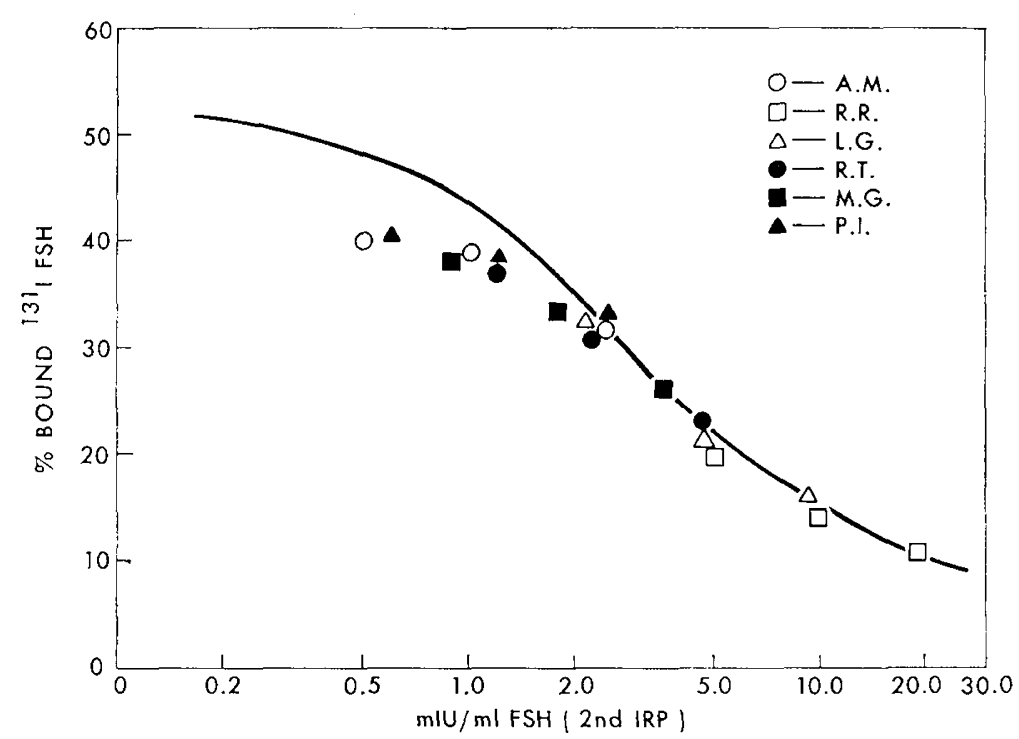

Fig. 1. Comparison of multiple dilutions carried out on unknown samples of kaolin-acetone urinary concentrates and the standard material, 2nd IRP. Note deviation from parallelism which occurs between 1-2 mIU of FSH per ml (semilog plot). 
sample was first centrifuged; the supernatant was then removed and the $\mathrm{pH}$ was adjusted to 4.5 with glacial acetic acid. Approximately $0.6 \mathrm{~g}$ kaolin was added and stirred thoroughly. The mixture was centrifuged, the supernatant was discarded, and the kaolin button was resuspended in 60-90 $\mathrm{ml}$ tap water containing 2-3 drops glacial acetic acid. Following a third centrifugation, $3.0 \mathrm{ml} 2 \mathrm{~N}$ ammonium hydroxide were added and the kaolin was thoroughly stirred with a Vortex mixer. After centrifugation, the supernatant fluid was removed to a $15-\mathrm{ml}$ test tube and the kaolin was washed with $1.5 \mathrm{ml}$ distilled water. The water eluate was then added to the ammonium hydroxide (total 4.5 $\mathrm{ml}$ ) and the $\mathrm{pH}$ was adjusted to 5.5 with glacial acetic acid. Following acidification, $9.0 \mathrm{ml}$ acetone were added and mixed, and the tube was allowed to stand $30 \mathrm{~min}$ or more. The precipitate was then centrifuged, the supernatant was discarded, and the resultant button was suspended in $2.0 \mathrm{ml}$ distilled water (Vortex mixer). The final suspension was centrifuged until clear and the supernatant fluid was introduced into the radioimmunoassay in amounts of 10-200 $\mu$ liters. Multiple dilutions were performed on every sample reported in this study. A control tube containing no first antibody was prepared for each unknown and run in the assay in order to check for nonspecific binding.

\section{Patients}

All patients were hospitalized for evaluation of either short stature or delayed adolescence. Informed consent was obtained from the parents of all individuals participating in the study. A total of 13 prepubertal children-11 boys and 2 girls-between ages 4 and 17 , were given clomiphene in daily doses of $0.1 \mathrm{mg}$ to $0.001 \mathrm{mg}$ for one week. Thirteen pubertal subjectseleven boys and two girls-between ages 12 and 17 were given the same drug in daily doses ranging from 100 to $0.1 \mathrm{mg}$. Sequential studies over 12-16 months were carried out in two of the individuals reported.

Six of the twenty-six patients were diagnosed as having isolated growth hormone deficiency, the remainder being essentially normal. Urine collections were made while the subjects were studied in the Pediatric Clinical Research Center. Twenty-four hour creatinine determinations were carried out on all samples to insure completeness of collections. There were no untoward effects from clomiphene experienced by any of these young individuals.

\section{Results}

\section{Radioimmunoassay of Urinary Concentrates}

As shown in Figure 1, deviation from parallelism between unknown and standard may occur at levels of the assay considered utilizable if highly purified material is employed. Thus, a potency estimate determined from a single dose response near the limits of detection may not be valid. Attention to serial dilutions (and thus the determination of sensitivity for that particular unknown) is of prime importance in insuring that the unknown is behaving in a fashion similar to standard, particularly if suppression below the low levels of gonadotropins present in the urine of children is investigated. Because parameters of assay sensitivity may be different for a given biologic specimen and a highly purified standard material, a stated assay sensitivity in terms of purified standard may not bear relevance to the actual sensitivity of the assay as applied to biologic samples. The 2nd IRP was utilized as reference material in all assays, and deviation from parallelism usually occurred at $1.0-1.5 \mathrm{mIU} / \mathrm{ml}$ (Fig. 1) in both FSH and $\mathrm{LH}$ radioimmunoassays.

A laboratory urine standard, made by the above concentrating technique, was run in multiple dilutions on each FSH and LH radioimmunoassay. Utilizing urinary material, the maximum within-assay coefficient of variation was $6 \%$ for $\mathrm{LH}$ and $10 \%$ for FSH. Betweenassay coefficient of variation was $18 \%$ for $\mathrm{LH}$ and $30 \%$ for FSH. All urine samples from a given patient were run on the same assay.

\section{Data Evaluation}

Because of differences between the base line levels of various subjects as well as considerable variation for values within a given individual, the data do not readily lend themselves to classical metric analysis. However, if the results of all hormone determinations are compared between the different treatment groups, using an ametric statistical test, distinguishing features of a given group may become apparent.

The use of the sign test [4] allows such a comparison by considering whether a given mean result did or did not change in a given direction. The present data could thus be viewed by determining whether mean pre- and post-treatment values went up, down, or remained the same, irrespective of the amount of change. This type of analysis eliminates the need to quantitate suppression but does allow recognition of a unique group of responses. 
If the mean values pre- and postclomiphene treatment are evaluated by this method for FSH, LH, and testosterone (considering the changes in each hormone as an independent observation) in the four prepubertal groups (Fig. 2, $A-D$ ) and the four early pubertal groups (Fig. 3, $A-D$ ), significance for downward trends is obtained only in the early pubertal patients who received $100 \mathrm{mg}$ clomiphene per day $(P<0.01)$. Thus clomiphene seems to have effected a significant suppression of the measured hormones when administered to early pubertal subjects only at the $100 \mathrm{mg}$ per day dose; the seven remaining treatment goups (see below) do not show a statistically significant change by these means.

\section{Prepubertal Subjects}

We have previously reported suppressive effects on urinary FSH and plasma testosterone when $1-100 \mathrm{mg} /$ day of clomiphene were given to a prepubertal child for 1 week [9]. Figure 2, $A-D$, illustrates the results when 0.1 to $0.001 \mathrm{mg} /$ day of clomiphene was given for a similar time period to sexually immature children.

Two of five patients given $0.1 \mathrm{mg} /$ day of clomiphene suppressed urinary FSH to undetectable levels. A third individual did depress his plasma testosterone (from 43 to $15 \mathrm{mg} / 100 \mathrm{ml}$ or less) and FSH was also lowered from base line values. Thus, two or possibly three of five children may have exhibited suppressive effects of clomiphene when as little as $0.1 \mathrm{mg} /$ day of the drug was administered.

At doses of 0.01 and $0.001 \mathrm{mg} /$ day taken for 1 week, no changes in either urinary FSH or plasma testosterone were demonstrated (Fig. 2, $B-D$ ). Two boys were given the drug at the $0.01 \mathrm{mg}$ dose for 16-18 days, and one individual displayed a downward trend in urine FSH levels by the end of 1 week of treatment. There were no stimulatory type responses observed at any dose level in any of the prepubertal patients.

\section{Early Pubertal Subjects}

In order to test whether the early pubertal individual (approximately stage 2 of Marshall and Tanner for pubic hair and genitalia or breast development [14, 15]) also exhibited a fall in gonadotropins and testosterone following clomiphene administration, three boys were given $100 \mathrm{mg} /$ day for 1 week (Fig. $3 A$ ). Urinary LH as well as FSH could be easily measured in these more mature subjects. All three boys exhibited a downward shift in plasma testosterone, with a simi- lar gonadotropin trend in two. (See above under Data Evaluation.)

Of the four early pubertal patients given $10 \mathrm{mg} /$ day (Fig. 3B), one individual displayed a downward change in plasma testosterone; gonadotropins but not testosterone were depressed in the 17-year-old boy with a bone age of 14. One milligram of clomiphene daily (Fig. 3C) elicited a suppressive type response in a 17 year-old boy with delayed adolescence but did not cause any consistent hormonal changes in three other individuals who received this dose.

Finally, when $0.1 \mathrm{mg}$ clomiphene was given daily to four boys (Fig. 3D), neither stimulation nor depression of the hormones measured was demonstrated. It is of interest, however, that plasma testosterone seemed to rise in one of these patients.

\section{Midpubertal Subjects}

Three individuals in midpuberty (Tanner stage 3) were given $100 \mathrm{mg}$ clomiphene daily for 1 week, and two of the three (both girls) did have stimulatory LH responses (Figs. 4-6). The two females experienced the onset of menarche 17-24 days after drug treatment, demonstrating the significance of the urinary LH rise. One of these two girls was tested with $10 \mathrm{mg}$ clomiphene per day I year before the 100-mg course and no changes in urinary FSH or LH were obtained. The rise in gonadotropins between age 13 and 14 is of note and is consistent with the progression in secondary sexual characteristics.

The boy pictured in Figure 6 is of particular interest since he was tested on three occasions over a 16month period with $100 \mathrm{mg} /$ day of clomiphene. Although the first two tests carried out during early puberty showed suppressive type responses, the third test, performed during midpubertal development, resulted in no hormonal changes. The transition from suppressive responses to no change was accompanied by advancement of secondary sexual characteristics and increases in levels of both gonadotropins and testosterone.

\section{Discussion}

\section{Radioimmunoassay of Urinary Concentrates}

Although the radioimmunoassay of plasma gonadotropins has been an invaluable aid in increasing knowledge of the reproductive process, limitations in the use of this new technique are becoming apparent. 

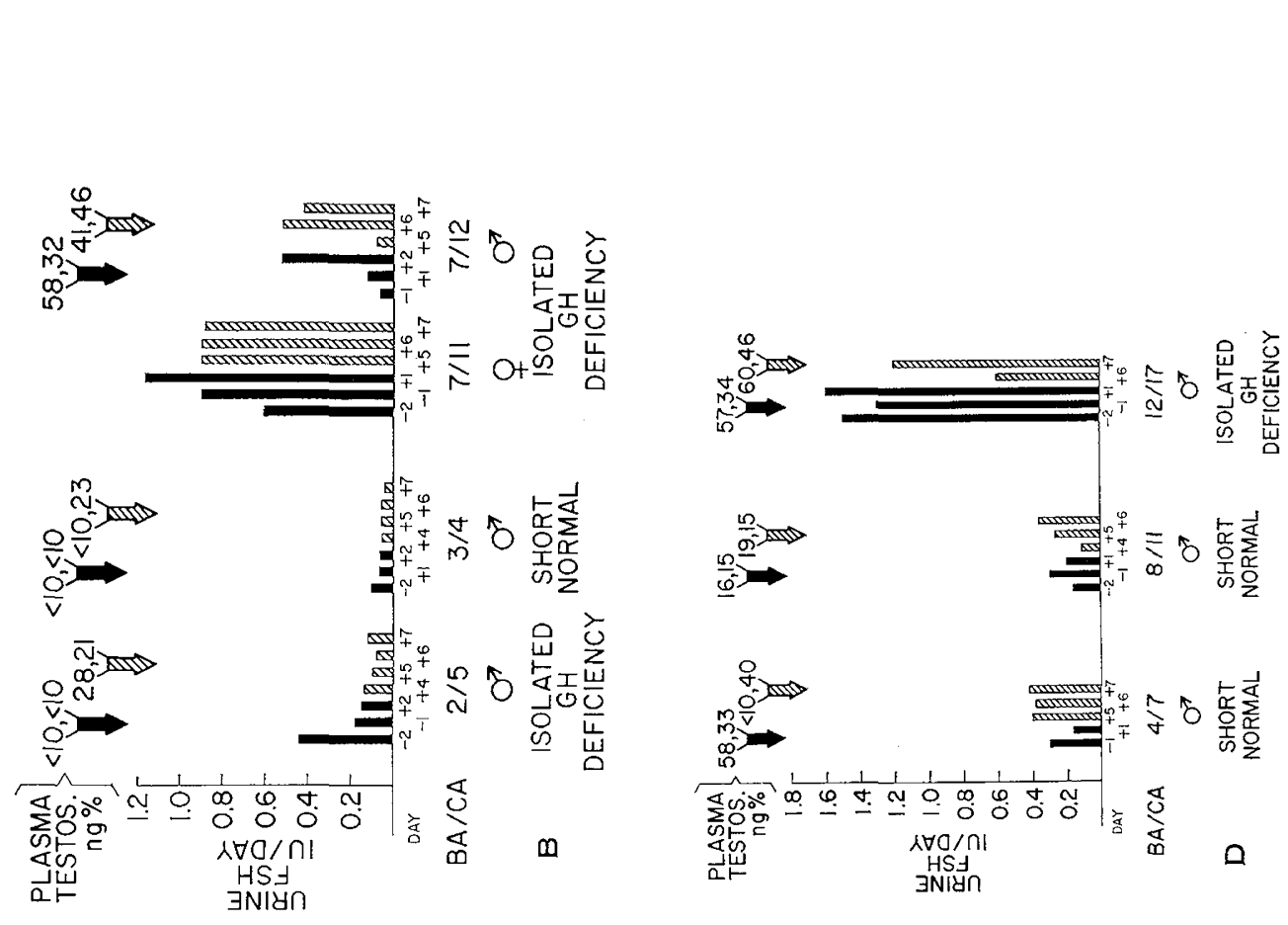

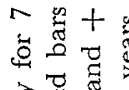

密要,

旅焉密

领起

0 .

Q

范节声

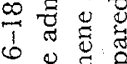
至 ㄴ.

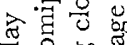

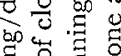

ㄷ.

$\ddot{0} \stackrel{3}{3}$ 事若:

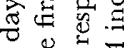
人吉志 융

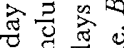
00 वे 0
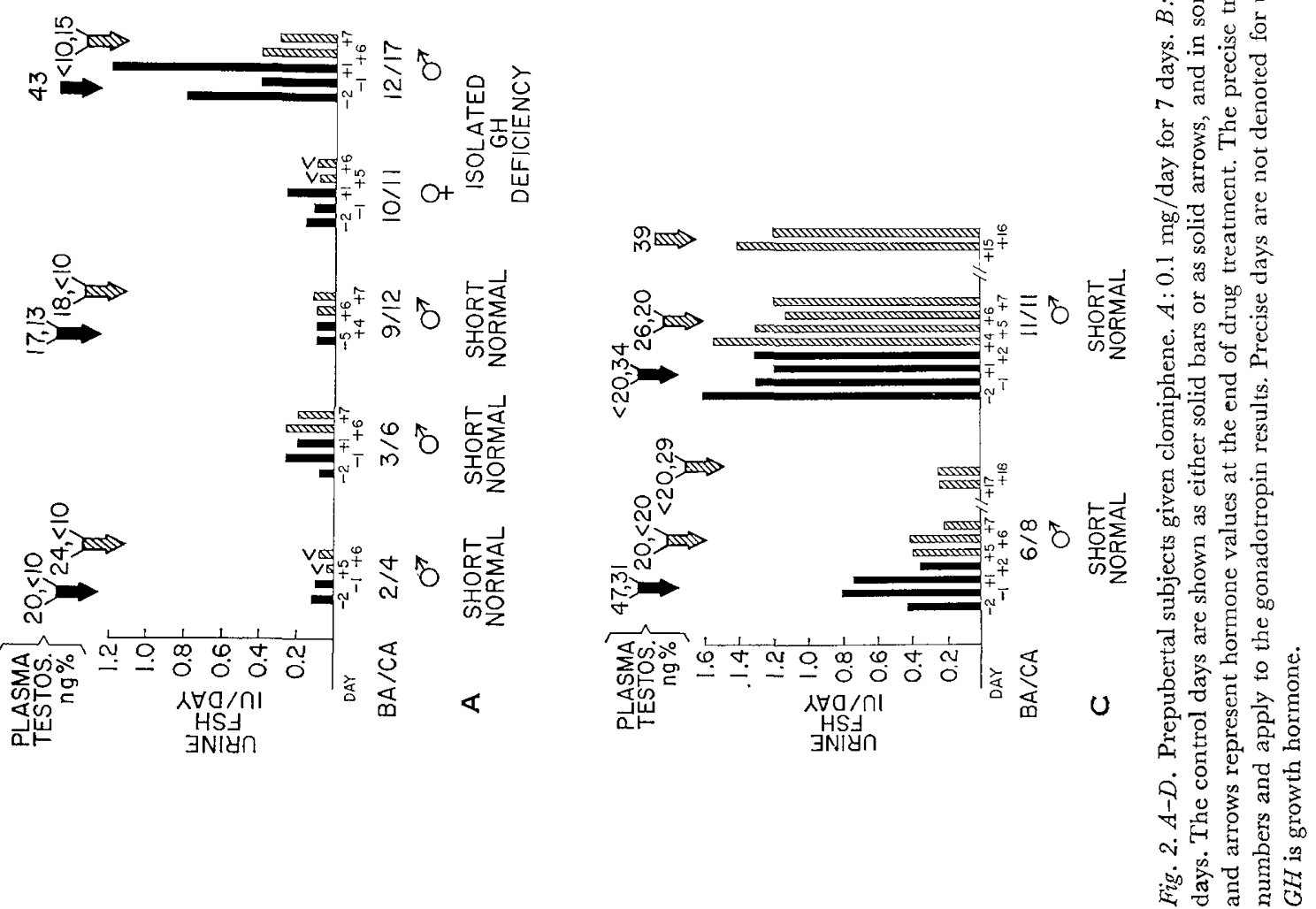

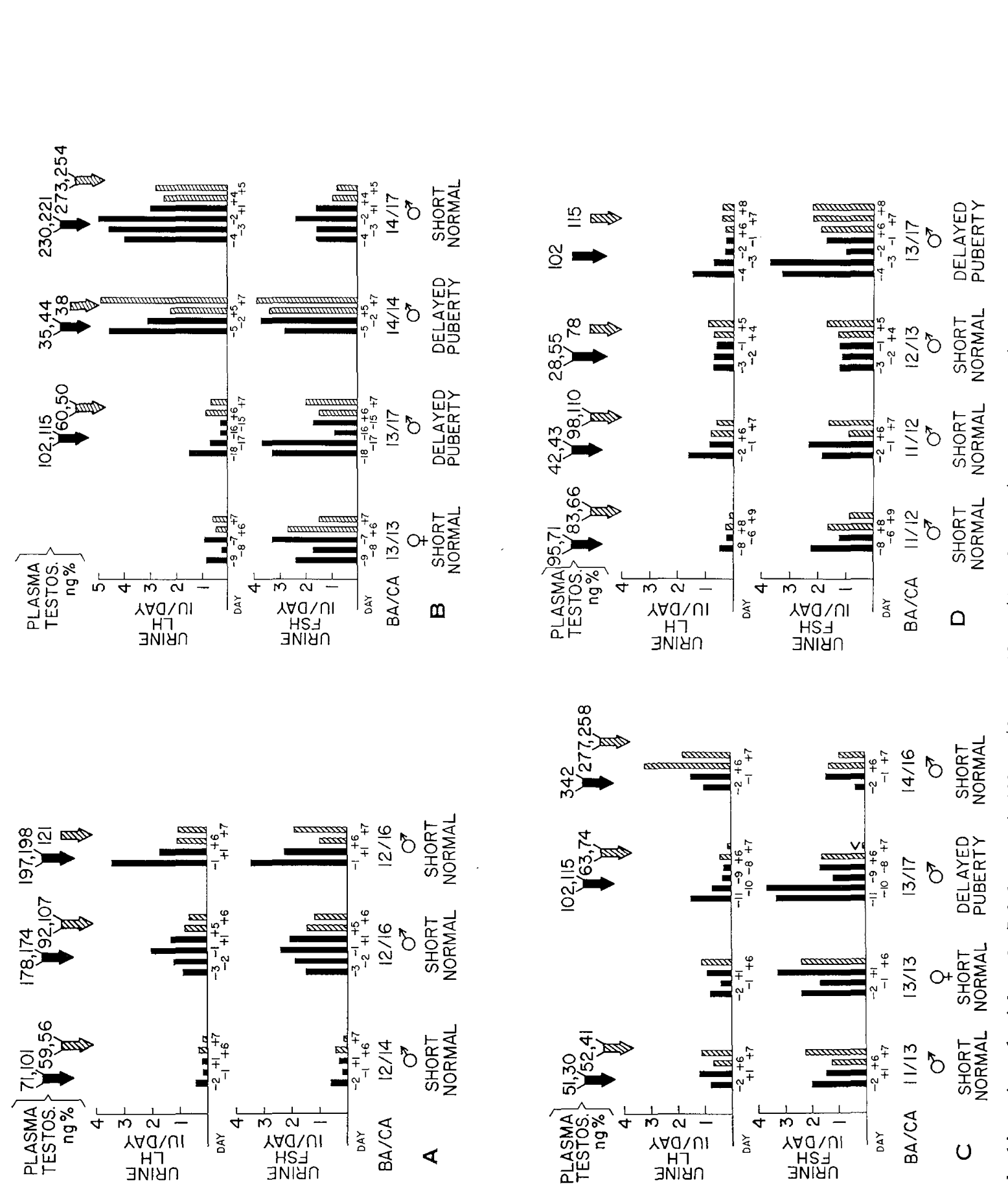

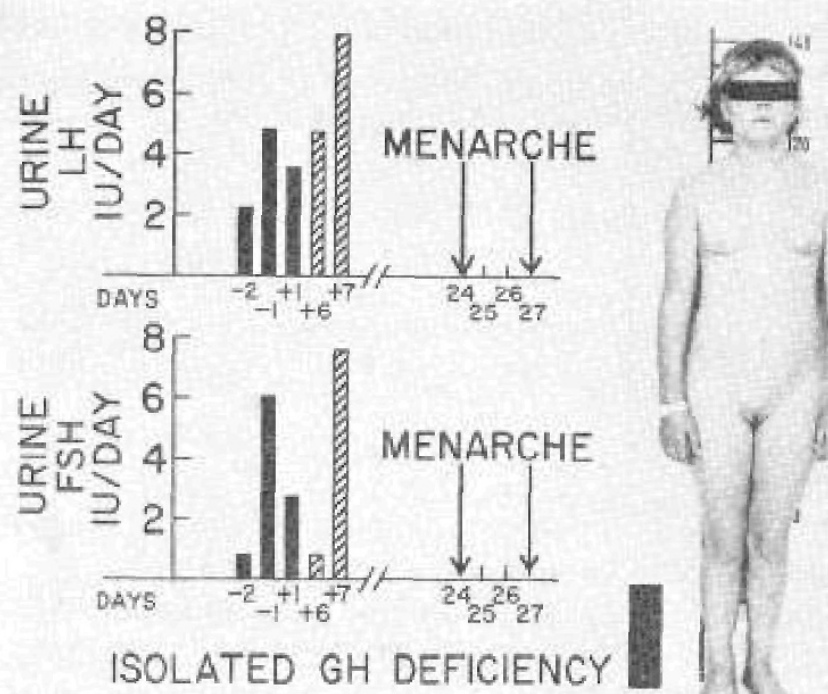

$B A / C A$

$12 / 14$

Fig. 4. Midpubertal girl given $100 \mathrm{mg}$ clomiphene per day for 1 week, with menses following 24 days after the onset of drug course. Note LH rise. See Figure 2 for codes.



Fig. 5. Sequential clomiphene study during female puberty. A 13-year-old early pubertal girl was given $10 \mathrm{mg} / \mathrm{day}$ of clomiphene for 1 week with no resultant gonadotropin changes. One year later, following an advance in secondary sexual characteristics, she was given 100 $\mathrm{mg}$ /day for a similar time period and exhibited a prompt rise in LH, as well as vaginal bleeding 17 days after the onset of treatment. No FSH stimulatory response was observed during the second clomiphene test despite the rise in gonadotropin excretion between ages 13 and 14. See Figure 2 for codes.

One of these limitations for plasma samples is sensitivity, and the assay of urinary concentrates may be one means of circumventing the problem. For example, the results of plasma specimens run on the above patients, as well as those in the previous study [9], could not detect suppressive changes in FSH and LH in prepubertal children. Furthermore, results from the radioimmunoassay of plasma have been unable to demonstrate 


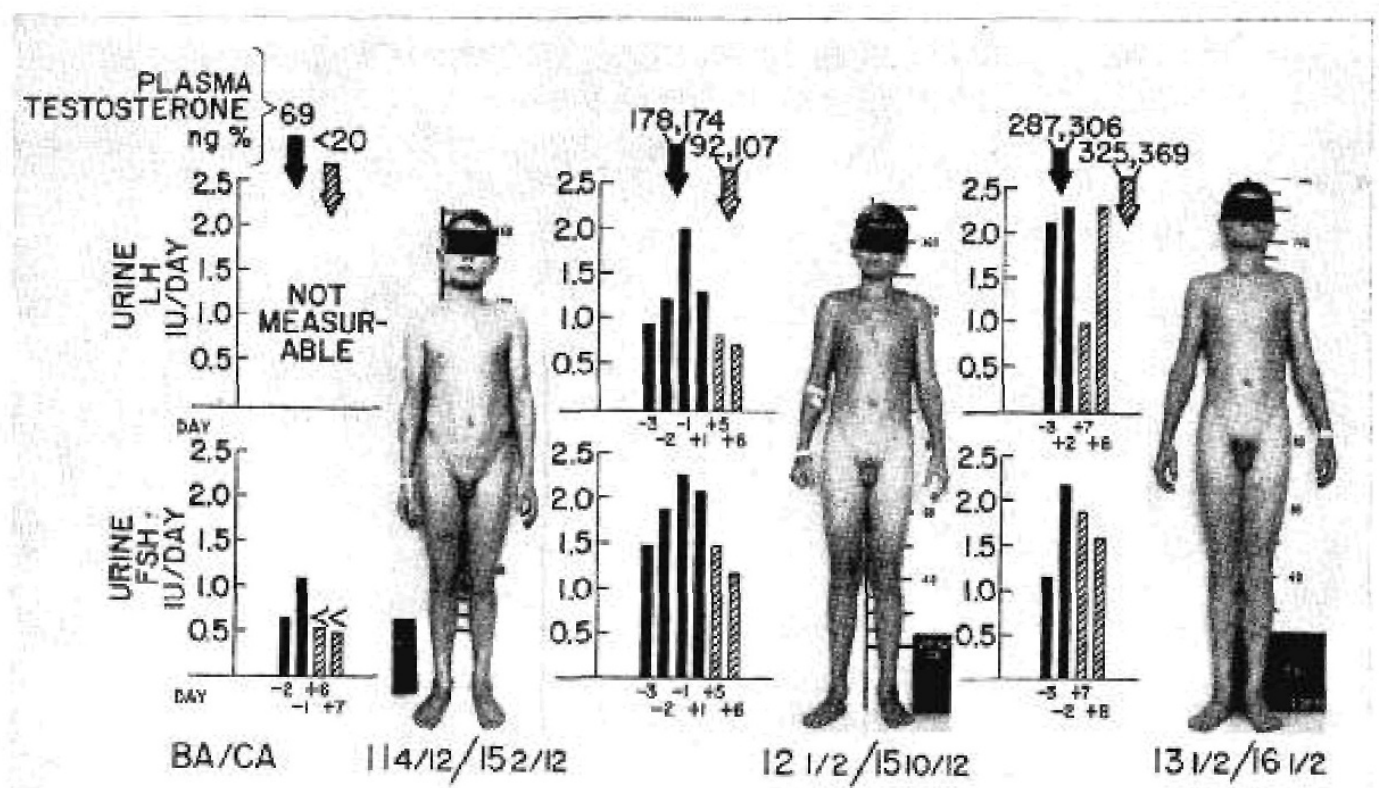

Fig. 6. Sequential clomiphene study during male puberty. A 15-year-old boy was tested on three occasions with $100 \mathrm{mg} /$ day of clomiphene given for 1 week. With advancing puberty, the values for testosterone and gonadotropins changed from an initial suppressive response to lack of suppression. Note the overall hormonal rises with advancing maturity. See Figure 2 for codes.

the wide differential of biologically determined urinary LH levels (>10-fold) which exists between the immature child and adult [18].

The major limitation in the use of urinary material is the considerable daily variation present. This variation has been noted previously in bioassay studies of urinary gonadotropins in both children and adults $[10$, 23]. Thus, adequate numbers of base line and experimental samples should be obtained.

The urinary levels of FSH and LH reported in this study are somewhat lower than both pooled biologically assayed values [18] and results obtained on 24-hr specimens by radioimmunoassay of either crude alcohol precipitates [16] or kaolin-acetone extracts [19]. Measurements of both urinary FSH and LH in adult males [13] obtained by the above techniques have also demonstrated values $10-50 \%$ lower than other published studies. The use of small aliquots of urine (i.e., $90 \mathrm{ml}$ ) rather than a total 24-hr specimen may have caused greater loss of hormone. However, every specimen from a given patient was concentrated at the same time and thus similar random loss from each sample would be expected.

Our strict attention to carrying out serial dilutions in each specimen could also contribute to the lower hormone values obtained. Thus, a single unknown point, read off a portion of the standard curve which is below the sensitivity for that particular unknown, would tend to give a falsely high estimate of hormone content.

A further problem in the present study is the difficulty of precisely defining suppression or stimulation. We have observed trends, and believe that useful information can be gleaned when a pattern is observed in the results of multiple hormone determinations (e.g., FSH and testosterone) or for a given hormone within a group of individuals. The sign test was useful in confirming the significance of these observations. The presence of an event such as vaginal bleeding is, of course, confirmatory of the $\mathrm{LH}$ rise observed in the two girls in midpuberty given $100 \mathrm{mg}$ clomiphene per day (Figs. 4 and 5).

\section{The Use of Clomiphene Citrate as a Weak Estrogen}

The present study was initially undertaken in an attempt to elicit a stimulatory gonadotropin response with clomiphene in the sexually immature child. When suppressive effects were found [9], we continued to work with this drug rather than switch to a pure estrogen compound. Clomiphene has both estrogenic and antiestrogenic activity and competes effectively for uterine cytosol receptors [8].

The biologic effect of clomiphene in causing breast hypertrophy in prepubertal boys given $100 \mathrm{mg}$ clomiphene for 1 week is also of note [9]. We have witnessed breast development in a castrated adult male given as 
little as $25-50 \mathrm{mg}$ clomiphene per day for 8 days. Thus, in the environment of low endogenous sex steroid, clomiphene can manifest significant estrogen-like activity.

\section{Clomiphene and Prepubertal Children}

The work presented here attempts to extend our previous studies [9] demonstrating suppressive type effects of clomiphene. If the prepubertal gonadal hypothalamic negative feedback mechanism in children is both operative and highly sensitive, and, if clomiphene acts in the adult as a steroid antagonist at the hypothalamic level [3], then reducing the dose of clomiphene might be expected to elicit a stimulatory gonadotropin response in the prepubertal child. This hypothesis was tested by lowering the dose of clomiphene below the suppressive level in an attempt to obtain a ratio of estrogen antagonist to hypothalamic receptors which would mimic the adult (and thus cause stimulation of gonadotropins). Our inability to achieve such stimulation raises the question as to whether clomiphene is indeed acting as a steroid antagonist in the adult.

Clomiphene suppression occurred at $500 \mathrm{mg} /$ day in a normal adult male undergoing a 20-day sequential study in which stimulation took place at doses ranging between 25 and $200 \mathrm{mg} /$ day [12, 25]. Thus, the difference in suppressive effects of clomiphene between children and adults is probably greater than 500 -fold (assuming adults require $500 \mathrm{mg} /$ day or more for suppression and prepubertal children suppress at $1.0 \mathrm{mg} /$ day). The large differences in suppressive doses between adult and child is in sharp contrast to data in the rat, in which the prepubertal hypothalamus is only 3-5 times more sensitive to estrogen feedback than that of the adult [17].

\section{Clomiphene and Pubertal Children}

Since $100 \mathrm{mg}$ clomiphene (but not lower doses) suppressed gonadotropins in the early pubertal patients, inordinate hypothalamic sensitivity to steroid feedback may extend into the early stages of sexual maturation. Additionally, the one boy followed sequentially into midpuberty took between 8 and 16 months before his increased hypothalmic sensitivity to clomiphene diminished. Still, he did not manifest a stimulatory response at age 16.5 and presumably needed even further sexual maturation for this type of effect.

The two girls who exhibited stimulatory LH responses to $100 \mathrm{mg}$ clomiphene suggest that this ability is attained at least by midpuberty in the female. Perhaps the development of positive feedback potential to estrogen, suggested by the preovulatory LH surge of mature women [20], is acquired only in midpuberty. The acquisition of positive feedback by estrogen may be directly related to the stimulatory response to clomiphene attained during puberty. It remains to be established whether the estrogenic action of clomiphene is playing a role in the ability of the drug to elevate gonadotropin levels in the adult.

The results of animal studies [22] are consistent with a multistaged progression of puberty, such as is suggested by the data presented herein. Changing sensitivity of hypothalamic receptors to steroids may be associated only with the initiative processes. A later event could then be the onset of estrogen-induced gonadotropin release. Such a scheme is attractive, but it remains hypothetical, at least for the human.

\section{Conclusion}

The inability to stimulate urinary gonadotropins and plasma testosterone in prepubertal and early pubertal children with clomiphene citrate is of particular note. The use of clomiphene has been proposed as a test of pituitary-gonadal interaction in both men and women $[3,7]$. Although a significant stimulatory hormonal response from the drug precludes a diagnosis of hypogonadotropism, the lack of a stimulatory effect does not help to confirm the diagnosis of hypogonadotropism unless the (bone) age of the patient assures sufficient (i.e., midpubertal) maturation of the neural centers which are necessary for the adult type response.

\section{References and Notes}

1. Albert, A.: Human urinary gonadotropins. Recent Progr. Hormone Res., 12: 227 (1957).

2. August, G. P., Tkachuk, M., and Grumbach, M. M.: Plasma testosterone-binding affinity and testosterone in umbilical cord plasma, late pregnancy, prepubertal children and adults. J. Clin. Endocrinol. Metab., 29: 891 (1969).

3. Bardin, G. W., Ross, G. T., ANd Lipsett, M. B.: Site of action of clomiphene citrate: a study of the pituitary-leydig cell axis. J. Clin. Endocrinol. Metab., 27: 1558 (1967).

4. Bross, I. D.: Statistical criticisms. Cancer, 13: 394 (1960).

5. Burr, I. M., Sizonenko, P. C., Kaplan, S. L., and GrumBACH, M. M.: Hormonal changes in puberty. I. Correlation of serum luteinizing hormone and follicle stimulating hormone with stages of puberty, testicular size, and bone age in normal boys. Pediat. Res., 4: 25 (1970).

6. Faiman, C., Ryan, R. J., and Albert, A.: FSH and LH activity in urinary extracts: a comparison of radioimmunoassay and bioassay methods. J. Clin. Endocrinol. Metab., 28: 1076 (1968).

7. Jacobsen, A., Marshall, J. R., Ross, G. T., and Cargille, 
C. M.: Plasma gonadotropins during clomiphene induced ovulatory cycles. Amer. J. Obstet. Gynecol., 102: 284 (1968).

8. Korenman, S. G.: Relation between estrogen inhibitory activity and binding to cytosol of rabbit and human uterus. Endocrinology, 87: 1119 (1970).

9. Kulin, H. E., Grumbach, M. M., and Kaplan, S. L.: Changing sensitivity of the pubertal gonadal hypothalamic feedback mechanism in man. Science, 166: 1012 (1969).

10. Kulin, H. E., Rifkind, A. B., Ross, G. T., And Odell, W. D.: Total gonadotropin activity in the urine of prepubertal children. J. Clin. Endocrinol. Metab., 27: 1123 (1967).

11. Kulin, H. E., Rifkind, A. B., And Ross, G. T.: Human luteinizing hormone (LH) activity in processed and unprocessed urine measured by radioimmunoassay and bioassay. J. Clin. Endocrinol. Metab., 28: 543 (1968).

12. Kulin, H. E., Weinstein, R. L., Kaplan, S. L., and GrumBACH, M. M.: The effect of high and low dose clomiphene on urinary gonadotropins and plasma testosterone in normal and XXY adult males and prepubertal children (Abstract). Fifty-Second Meeting of The Endocrine Society, St. Louis, Mo., p. 58, 1970.

13. Kulin, H. E., Weinstein, R. L., Kaplan, S. L., and GrumBACH, M. M.: Differential FSH and LH suppression by Iow dose estrogen in normal adult males (In preparation).

14. Marshall, W. A., And Tanner, J. M.: Variations in pattern of pubertal changes in girls. Arch. Dis. Childhood, 44: 291 (1969).

15. Marshall, W. A., and Tanner, J. M.: Variations in the pattern of pubertal changes in boys. Arch. Dis. Childhood, 45: 13 (1970).

16. Rartr, S. M. B., Light, C., AND Blizzard, R. M.: Urinary follicle-stimulating hormone excretion in boys and adult males as measured by radioimmunoassay. J. Clin. Endocrinol. Metab., 29: 884 (1969).

17. Ramirez, D. V., and McCann, S. M.: Comparison of the regulation of luteinizing hormone $(\mathrm{LH})$ secretion in immature and adult rats. Endocrinology, 72: 452 (1963).

18. Rifkind, A. B., Kulin, H. E., ANd Ross, G. T.: Follicle stimulating hormone $(\mathrm{FSH})$ and luteinizing hormone $(\mathrm{LH})$ in the urine of prepubertal children. J. Clin. Invest., 46: 1925 (1967).

19. Rifkind, A. B., Kulin, H. E., Cargille, C. M., Rayford, P. C., AND Ross, G. T.: 24 hour urinary luteinizing hormone (LH) and follicle stimulating hormone (FSH) excretion in normal children. J. Clin. Endocrino1. Metab., 31: 517 (1970).

20. Ross, G. T., Cargille, C. M., Lipsett, M. B., Rayford,
P. L., Marshall, J. R., Strott, C. A., and Rodbard, D.: Pituitary and gonadal hormones in women during spontaneous and induced ovulatory cycles. Recent Progr. Hormone Res., 26: 1 (1970).

21. Sizonenko, P. C., Burr, I. M., Kaplan, S. L., And Grumbach, M. M.: Hormonal changes in puberty. II. Correlation of serum luteinizing hormone and follicle stimulating hormone with stages of puberty and bone age in normal girls. Pediat. Res., 4: 36 (1970).

22. Smith, E. R., ANd Davidson, J. M.: Role of estrogen in the cerebral control of puberty in female rats. Endocrinology, 82: 100 (1968).

23. Stevens, V. C.: Discrepancies and similarities of urinary FSH and LH patterns as evaluated by different assay methods. In: Immunoassay of Gonadotropins, p. 338. (Karolinska Symposia on Research Methods in Reproductive Endocrinology, First Symposium, Stockholm, 1969).

24. Stevens, V. C.: Comparison of FSH and LH patterns in plasma, urine, and urinary extracts during the menstrual cycle. J. Clin. Endocrinol. Metab., 29: 904 (1969).

25. Wernstern, R. L., Kulun, H. E., Kaplan, S. L., and Grumbach, M. M.: Hypothalamic dysfunction in Klinefelter's syndrome: gonadotropin suppression by clomiphene (In preparation).

26. We thank the National Pituitary Agency, National Institutes of Health, and the British Medical Research Council for purified FSH, FSH antiserum, and 2nd IRP. Appreciation is also expressed to Dr. R. L. Weinstein, Dr. David Rodbard, M. Tkachuk, I. Holst, A. Jardiolin, K. Hayward, and the physicians and nurses of the Pediatric Clinical Research Center.

27. Presented in part at the Fifty-second Meeting of The Endocrine Society, St. Louis, Mo., June, 1970.

28. Dr. Kulin was on out-of-service training from the National Institute of Child Health and Human Development, National Institutes of Health. Dr. Kaplan was the recipient of a Research Career Development Award, the National Institute of Child Health and Human Development.

29. Supported in part by grants from the National Institutes of Child Health and Human Development and the National Institute of Arthritis and Metabolic Diseases, National Institutes of Health.

30. Requests for reprints should be addressed to: Howard E. KuLIN, M.D., 10B-09, Reproduction Research Branch, National Institute of Child Health and Human Development, National Institutes of Health, Bethesda, Md. 20014 (USA).

31. Accepted for publication June 18, 1971. 